\title{
Text and video mining solutions to national security intelligence problems
}

\author{
A. Zanasi ${ }^{1} \&$ M. Artioli ${ }^{2}$ \\ ${ }^{I}$ ZANASI Alessandro Srl, Modena, Italy \\ ${ }^{2}$ Bridge 129 SPA, Reggio Emilia, Italy
}

\begin{abstract}
Since the end of the Cold War, the threat of large scale wars has been substituted by new threats: terrorism, organized crime, trafficking, smuggling, proliferation of weapons of mass destruction. The new criminals, especially the so-called "jihadist" terrorists, are using the new technologies, as those enhanced by Web2.0, to fight their war. But also intelligence analysts react in their daily fights using new technologies (e.g. text and video mining) to automatically analyze the content of information rich online data banks, suspected web sites, blogs, emails, chat lines, instant messages and all other digital media either for investigation (eg. to detect abnormal behaviors or links between people and organizations, trends of social and economic actions, topics of interest also if they are "sunk" among terabytes of information) or understanding (eg. in radicalization studies). This paper presents an integration of text and video mining in applicative examples.
\end{abstract}

Keywords: text mining, video mining, national security, intelligence.

\section{National security, intelligence, technology}

\subsection{Netwar terrorism threats}

1. After the $9 / 11$ shock, the world is requiring a different intelligence: open to several sources (especially the "open sources"); open to strong utilization of new information technologies to take profit of the information (often contradictory) explosion (information density doubles every 24 months and its costs are halved every 18 months [1]); open to the contributions of the best experts, also outside government [2], e.g. through a public-private partnership (PPP or P3: a system in which a government service or private business venture is funded and 
operated through a partnership of government and one or more private sector companies).

2. New terrorists are typically organized in small units and coordinate their activities on line. This phenomenon has been called Netwar (a form of conflict marked by the use of network forms of organizations and related doctrines, strategies, and technologies) [3] experiencing a paradigm shift from an organization-driven threat architecture (i.e., communities and social activities focused around large companies or organizations) to an individual-centric threat architecture (increased choice and availability of opportunities focused around individual wants and desires).

3. Due to this growing of virtual communities, a strong interest towards the capability of automatic detection and evaluation of content of communications exchanged inside these communities is also growing.

Seen that too many web sites disseminate the al Qaeda leadership's messages [4], automatic retrieval and analysis of texts and videos, understanding terrorists' cultural backgrounds-skills may be done thanks to text and video mining technologies [5, 6]. For an introduction to data mining: [7], to text mining technology and its applications to intelligence: [8], to video mining: [9].

\subsection{Intelligence and its application fields}

Today's intelligence analysts face different applicative areas where data, text and video mining may be applied:

\subsubsection{Marketing}

To learn more about customers and their wishes and needs, applying text mining to emails, chats, blogs [10] or video mining to capture the movement of people through a shop, in order to spot shoplifting activity and /or observe the order in which people browse through the store.

\subsubsection{Strategy}

To understand what competitors are doing and what they will do in the future, analyzing automatically their patents, their executives' declarations, the press surveys (for competitive intelligence [8, 11]).

\subsubsection{National security}

To monitor the web (to protect our citizens $[12,13]$ ) and the reality (through video surveillance systems coupled with video mining) to prevent terrorist attacks.

\subsection{Understanding the enemy and the radicalization process}

1. After 9/11 attack in NYC, 3/11 in Madrid, 7/7 in London and other ones, we are asking ourselves why quiet boys, eventually born in western countries commit such horrible acts, i.e. why they radicalize their thoughts and actions. One of the defining features of cultural studies applied to the study of violent radicalisation is the prominence this leaves to language: do the concepts used affect or even determine perceptions of the adversary and thus consequently 
public policy? Does language "construct" interests or is it merely reflecting them?

A significant amount of terror acts are committed against symbolic targets: how warfare on symbols functions and how it affects public opinion, government behaviour and terrorist-supporter's opinion?

The capability of analyzing the written texts is necessary to understand the reasons behind the terrorists' acts.

2. Furthermore, one of the more popular ways of communications from terrorists to target audiences is through the use of so called suicide videos (or martyrdom operations, as Hamas call them) or videos showing beheadings or executions and through written texts filled with religious or pseudo-religious metaphors, usually followed by direct threats.

3. So a particular attention must be dedicated to analysis of these texts and videos by automatic tools as text and video mining (seen that their volume is so huge to make impossible to human beings to detect and analyze all of them manually).

\section{The team}

\subsection{ZANASI Alessandro Srl}

ZANASI Alessandro $\mathrm{Srl}$ is an advisory company serving security research market, focusing on technology application to intelligence. It helps its clients build, implement and develop successful strategies in security research and, if necessary, be funded by private or public capital.

The founder was appointed ESRAB member in 2005 and, in 2007, ESRIF member.

\subsection{Bridge129}

Bridge 129, founded in 2000 with HQs in Reggio Emilia (Italy), is focused on Osint Solutions development.

Its background is in computer vision, video management for security, CCTV. After some years it is specializing in data retrieval (from video and from texts), and in chats, social networks, multimedia analysis (through video and text mining).

Bridge experience in video analysis is now considered an asset for the fight against terrorism.

\section{Technology}

\subsection{Video mining}

Video Mining seeks to automate what is now a very tedious, generally humanpowered process of reviewing video for content that is potentially of intelligence value from two points of view: 
- Computer vision and machine learning topics such as

○ Object detection, tracking, event detection and understanding

- Scene classification, recognition and modeling

- Intelligent content services such as indexing, video browsing, summarization, content browsing and change detection

- Application of these techniques to

- Seeking to automate processing of surveillance cameras to determine anomalous behavior and to

○ Retrieve events such as bombings or beheadings.

\subsection{Extraction of moving objects from the scene and their classification}

The segmentation of the movement aims to determine the regions where objects are (people or vehicles) in motion. The extraction of the movement can focus the attention of subsequent processes, such as tracking and analysis of behavior, only on those regions. Many methods of segmentation use space and time information over image sequences. We summarize briefly the different approaches to the segmentation of the movement:

1) Subtraction of the background. It is the most widely used technique for extracting the movement. We recognize the regions in motion by a subtraction pixel for pixel between the frame and image of the background.

2) Time difference. This technique, to extract the motion, makes the difference between two or more consecutive frames in a sequence.

3) Optical Flow. The methods based on optical flow can be used to determine moving objects in the context of camera-motion.

In addition to the basic methods previously cited there are several more complex background models. The most common is the Mixture of Gaussians which classifies the value of each pixel in three Gaussians distributions, matching the background, foreground and shadows; also updates the components of the mixture according to the probability of belonging to each class. So objects that move slowly are well treated.

It is necessary to properly classify objects to apply the tracking and analysis of the behavior. The approaches to the classification of moving objects are grouped into two categories: classification based on shape, and classification based on movement.

\subsection{Text retrieval from video}

Several techniques exist to extract text in easy situations and software to perform OCR tasks is widespread in many commercial applications. Conversely, extracting textual information in natural images is a difficult task since the information may be affected by strong noise or hidden to be filtered out by robust pattern recognition techniques. Several techniques may be applied that involve trained classifiers such as the Support Vector Machines to discriminate whether an image patch may contain textual information. 
Text extraction can be used to obtain information from images covering data like car plates, headlines comments and text on objects.

\section{Examples}

\subsubsection{APR-automatic plate recognition}

The number plate recognition basically requires much more work and attention compared to headlines comments. In effect any overlapped data in an image has evident no correlation with image background and it's easy to retrieve and filter out.

On the other side the extraction of data within image/video is dependent to image environment and no often easy to extract. I.e. the plate of a car can be affected by light reflections, dust and so on. In this case text retrieval applicability really depend on video source quality.

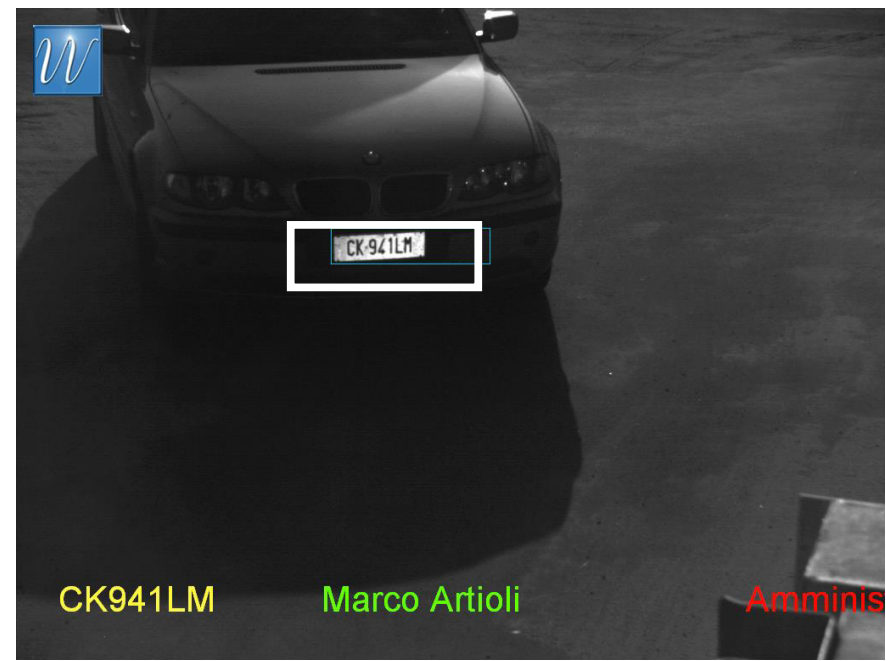

Figure 1: APR example.

The image refers to an outdoor camera installation in daytime with sun against.

The software acts like a plate detector and a number plate recognition. The plate in effect is found and bounded via a blue rectangle. The algorithm search inside this limited area the characters (segmentation process) and yield the plate read.

\subsubsection{Smoke, fire and weapons detection}

Autonomous and automatic videosurveillance system, capable of independently detecting the presence of smoke or flames in a video stream from a fixed camera has been achieved by emulation of artificial processes of visual human observation.

Once detected the presence of smoke and/or flame an alarm is generated and sent via e-mail, sms, mms with the coordinates, image and so on. 


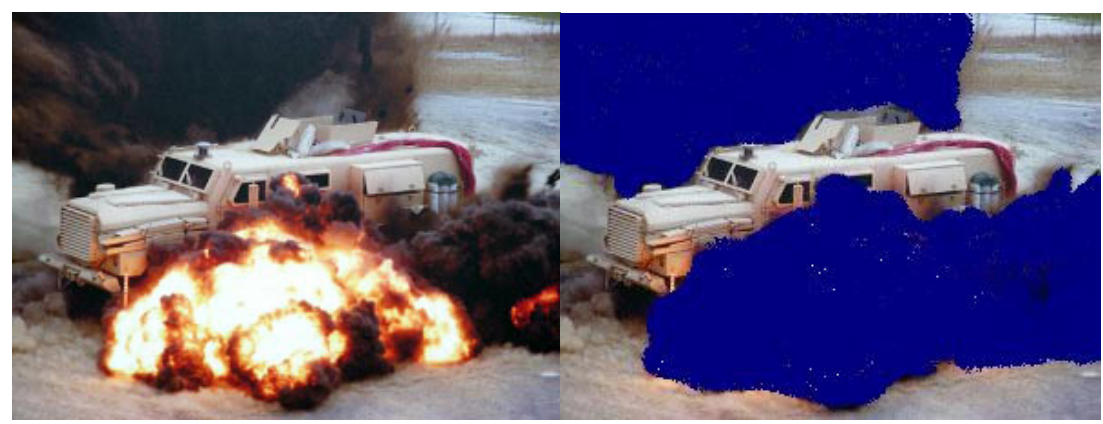

Figure 2: Truck explosion. Left frame refers to a truck explosion showing the real image. The action and smoke propagation is very fast. In the right frame a blue mask shows the algorithm detection. The detection speed is about one second.

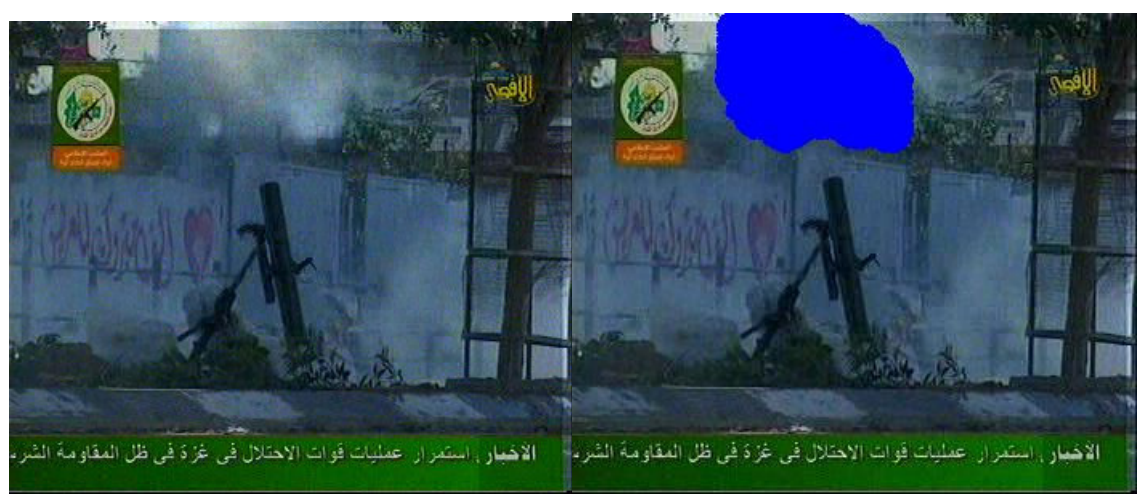

Figure 3: $\quad$ Smoke detection.

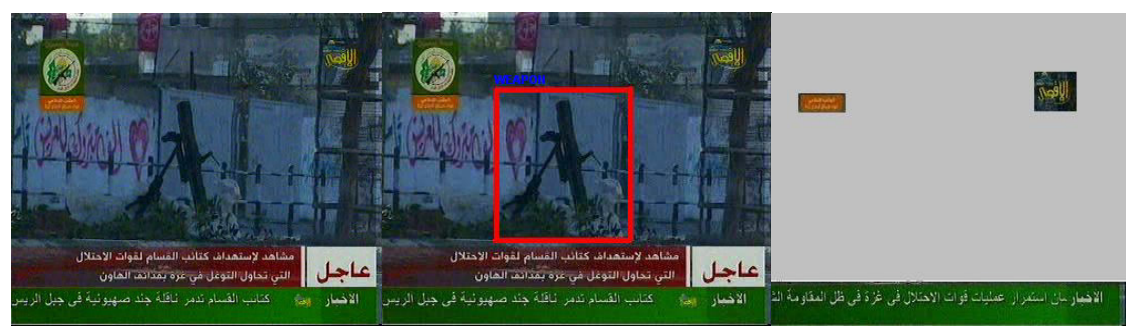

Figure 4: Weapon and text detection.

Video analysis Al-Aqsa TV, January 6, 2009. Also this video is taken outdoors and shows different components: weapon, smoke, text. Applying 
different algorithms we can detect either smoke or weapons or text (see the following paragraph).

The direction of algorithm implementation will follow the aim to support any intelligence activity. Keeping the Al Aqsa TV video as reference it is worthy to find the smoke source or investigate the presence of objects-weapons within the video source. Main limitations are based on real presence on video of the object to detect and noise due to overlapped features extraction in outdoor scenario.

\subsubsection{Arabic texts}

Applying the methods of text extractions we can filter from the scene the text on headlines comments (see Fig.4). Detection time is 1-3 seconds.

Once the text is retrieved and collected from the web, it may be automatically analyzed thanks text mining, extracting key concepts and/or clustering thematically coherent pages.

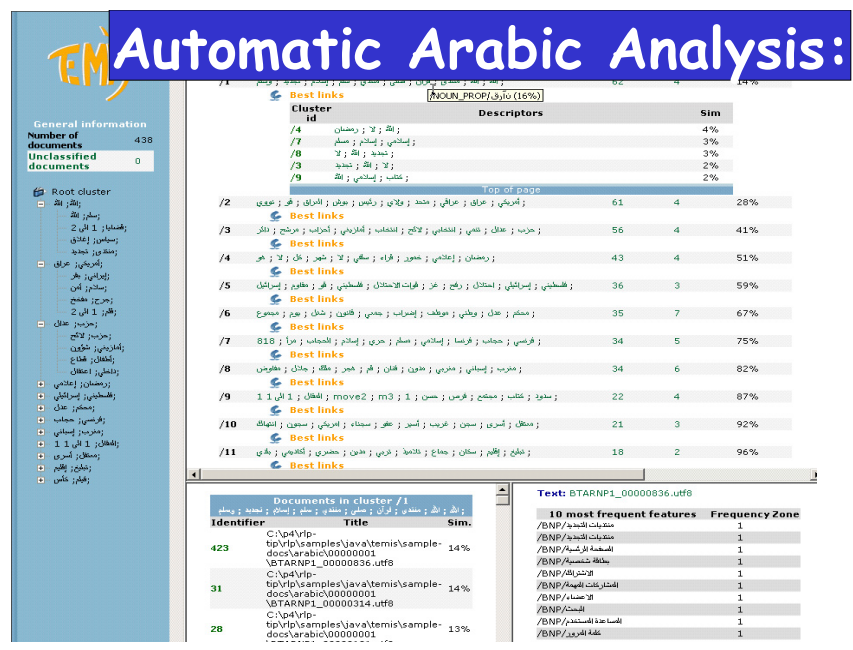

Figure 5: Text mining application.

\subsection{Biometric: face detection with SRI}

Techniques of SRI- Super Resolution Imaging are techniques that increase the resolution of an imaging system.

There are two approaches to these techniques: the passing of the diffraction limit of optics system or the trivial increasing of the signal-noise digital sensor.

One way to get pictures to super-high resolution is to merge together multiple images captured from the same position in order to visibly reduce the amount of digital noise introduced from the amplifier.

The algorithms used may be divided into two camps: those operating in the frequency domain, thanks to the Fourier Transform, and those working in the domain space, exploiting techniques such as segmentation or the study of the space of possible alignments for features of images. 
The increase of the resolution is usually done by creating a new image with a finer grid (lattice resolution) to go to put the pixels $\mathrm{N}$ extracted from original images, mediated. The HR of the missing pixels are interpolated with the most varied techniques, from simple linear interpolation algorithm to use the techniques being filtered rear.

With the techniques SRI increases the resolving power of the system and reduce the phenomenon of aliasing image LR (Low Resolution).

\subsection{Virtual communities monitoring}

A virtual community, whose blog and chats are typical examples, are communities of people sharing and communicating common interests, ideas, and feelings over the Internet or other collaborative networks. Howard Rheingold defined virtual communities as social aggregations that emerge from the Internet when enough people carry on public discussions long enough and with sufficient human feeling to form webs of personal relationships in cyberspace [14].

Most community members need to interact with a single individual in a oneto-one conversation or participate and collaborate in idea development via threaded conversations with multiple people or groups.

This type of data is, clearly, an exceptional source to be mined.

The first enemy of intelligence activity is the "avalanche" of information that daily the analysts must retrieve, read, filter and summarize. The Al Qaeda terrorists declared to interact among them through chat lines to avoid being intercepted [15]: interception and analysis of chat lines content is anyway possible and frequently done in commercial situations [16].

Using different text mining techniques it is possible to identify the context of the communication and the relationships among documents detecting the references to the interesting topics, how they are treated and what impression they create in the reader. The coupling of text and video mining applied to intelligence issues directed against the growing terrorist activity on the web [17] is a new weapon in the hands of law enforcement agents.

\section{European funded research}

\subsection{Reacting to the threat}

Today many threats to security are stateless in origin and transnational in scope. Terrorist groups have cells in multiple countries without the active support of any government but still capable of committing attacks with global impact. Potentially crippling attacks on the power grid or financial institutions could come from a computer anywhere in the world. Fighting elusive and transnational enemies requires international cooperation [18]. Technology has a central role in assuring protection of citizens: technology alone can't assure security but security without technology is not possible [19].

So an important aspect of fighting netwar terrorism is assuring international cooperation in developing new ideas and prototypes directed to protect populations against attacks. 
European Union, through the action of European Commission, since 2005, with the inception of ESRAB and then of ESRIF and with the funding assured through 7FP and other initiatives, has been active in warranting this cooperation among its academic and private scientists and researchers.

\subsection{ESRAB and ESRIF}

In 2005 a 'European Security Research Advisory Board' (ESRAB, including 50 high level strategists, nominated 'ad personam') [19] was created with the objective of defining the strategic missions, focus areas and priorities setting for security research programme. One of its results was the definition of Security theme in 7FP.

In 2007 a "European Security Research and Innovation Forum" ESRIF [20] was created with the objective of developing a mid and long term strategy for civil security research and innovation through public private dialogue. It should also trigger the investment of funds by the private sector in research strategic priorities, thereby complementing public investments. Moreover, it corresponds to the general aim of building a true European Research Area, notably by promoting greater coherence between investments in research and development allocated at European, national and regional levels.

\subsection{FP}

The Seventh Framework Programme for research and technological development (7FP) is the European Union's chief instrument for funding (through the mecanism of "Call for proposals") research over the period 2007 to 2013 where a specific line dedicated to Security theme was launched for the $1^{\text {st }}$ time in 2007 and whose budget amounts to a total of $€ 1.4$ billion.

New security and intelligence technologies (e.g. related to text and video mining) are expected to be funded principally for civil protection, bio-security, protection against crime and terrorism and to warrant intelligent surveillance and border security.

\section{References}

[1] Lisse W., The Economics of Information and the Internet, Competitive Intelligence Review, Vol.9 (4), 1998.

[2] Treverton G.F., Reshaping National Intelligence in an Age of Information, Cambridge University Press, 2001.

[3] Ronfeldt, D., Arquilla,J., The Advent of Netwar -Rand Corporation, 1996

[4] Riedel, B., Al Qaeda Strikes Back - Foreign Affairs, May/June 2007

[5] Kohlmann, E. The Real Online Terrorist Threat - Foreign Affairs, Sept/Oct.2006

[6] Mueller, J., Is There Still a Terrorist Threat? - Foreign Affairs, Sept/Oct 2006

[7] Cabena, P....Zanasi, A., 1998. Discovering Data Mining. Prentice Hall 
[8] Zanasi, A., (editor) Text Mining and its Applications to Intelligence, CRM and Knowledge Management - WIT Press: Southampton, Boston, 2007.

[9] Rosenfeld, A., et al., Video Mining, Kluwer Academic Publishers, 2003.

[10] Zanasi A, Email, chatlines, newsgroups: a continuous opinion surveys source thanks to text mining. Excellence in Int'l Research 2003 - ESOMAR (N1), 2003.

[11] Zanasi A., Competitive Intelligence Thru Data Mining Public Sources Competitive Intelligence Review, Vol.9(1), John Wiley \& Sons, Inc., 1998.

[12] Zanasi, A., New forms of war, new forms of Intelligence: Text Mining ITNS Conference, Riyadh 2007.

[13] Steinberg, J., in Protecting the Homeland 2006/2007 - The Brookings Institution, 2006

[14] Rheingold, H., The Virtual Community - MIT Press, 2000.

[15] The Other War, The Economist March $8^{\text {th }}$ Pag.26, 2003.

[16] Campbell, D., World under Watch, Interception Capabilities in the $21^{\text {st }}$ Century - ZDNet.co, 2001 (updated version of Interception Capabilities 2000, A report to European Parliament - 1999)

[17] Weimann, G., Terror on the Internet, US Institute of Peace Press, Washington, 2006

[18] Chertoff, M., The Responsibility to Contain -Foreign Affairs, Jan/Feb 2009

[19] Meeting the Challenge-The European Security Research Agenda Luxembourg: Office for Official Publications of the European Communities, 2006

[20] European security research and innovation in support of European security policies: Report -Luxembourg: Office for Official Publications of the European Communities-2008 